\title{
First muon-neutrino disappearance study with an off-axis beam
}

K. Abe, ${ }^{49, *}$ N. Abgrall,${ }^{16}$ Y. Ajima, ${ }^{18, \dagger}$ H. Aihara,${ }^{48, *}$ J. B. Albert,${ }^{13}$ C. Andreopoulos,${ }^{47}$ B. Andrieu, ${ }^{37}$ M. D. Anerella, ${ }^{6}$ S. Aoki,${ }^{27}$ O. Araoka,${ }^{18, \dagger}$ J. Argyriades,${ }^{16}$ A. Ariga,${ }^{3}$ T. Ariga,${ }^{3}$ S. Assylbekov,${ }^{11}$ D. Autiero, ${ }^{32}$ A. Badertscher,${ }^{15}$ M. Barbi, ${ }^{40}$ G. J. Barker ${ }^{56}$ G. Barr, ${ }^{36}$ M. Bass, ${ }^{11}$ M. Batkiewicz,${ }^{17}$ F. Bay, ${ }^{3}$ S. Bentham, ${ }^{29}$ V. Berardi, ${ }^{22}$ B. E. Berger, ${ }^{11}$ I. Bertram, ${ }^{29}$ M. Besnier, ${ }^{14}$ J. Beucher, ${ }^{8}$ D. Beznosko, ${ }^{34}$ S. Bhadra,${ }^{60}$ F. d. M. M. Blaszczyk, ${ }^{8}$ A. Blondel,${ }^{16}$ C. Bojechko, ${ }^{53}$ J. Bouchez, ${ }^{8,}$ S. B. Boyd ${ }^{56}$ A. Bravar, ${ }^{16}$ C. Bronner, ${ }^{14,28}$ D. G. Brook-Roberge,${ }^{5}$ N. Buchanan, ${ }^{11}$ H. Budd, ${ }^{41}$ R. G. Calland, ${ }^{30}$ D. Calvet, ${ }^{8}$ J. Caravaca Rodríguez, ${ }^{19}$ S. L. Cartwright, ${ }^{44}$ A. Carver,${ }^{56}$ R. Castillo, ${ }^{19}$ M. G. Catanesi, ${ }^{22}$ A. Cazes,${ }^{32}$ A. Cervera, ${ }^{20}$ C. Chavez, ${ }^{30}$ S. Choi,${ }^{43}$ G. Christodoulou, ${ }^{30}$ J. Coleman, ${ }^{30}$ G. Collazuol, ${ }^{24}$ W. Coleman, ${ }^{31}$ K. Connolly, ${ }^{57}$ A. Curioni, ${ }^{15}$ A. Dabrowska, ${ }^{17}$ I. Danko, ${ }^{38}$ R. Das, ${ }^{11}$ G. S. Davies, ${ }^{29}$ S. Davis,${ }^{57}$ M. Day, ${ }^{41}$ G. De Rosa, ${ }^{23}$ J. .P. A. M. de André, ${ }^{14}$ P. de Perio, ${ }^{51}$ T. Dealtry, ${ }^{36,47}$ A. Delbart, ${ }^{8}$ C. Densham, ${ }^{47}$ F. Di Lodovico, ${ }^{39}$ S. Di Luise, ${ }^{15}$ P. Dinh Tran, ${ }^{14}$ J. Dobson, ${ }^{21}$ U. Dore ${ }^{25}$ O. Drapier, ${ }^{14}$ T. Duboyski, ${ }^{39}$ F. Dufour, ${ }^{16}$ J. Dumarchez,${ }^{37}$ S. Dytman, ${ }^{38}$ M. Dziewiecki, ${ }^{55}$ M. Dziomba ${ }^{57}$ S. Emery, ${ }^{8}$ A. Ereditato, ${ }^{3}$ J. E. Escallier, ${ }^{6}$ L. Escudero,${ }^{20}$ L. S. Esposito, ${ }^{15}$ M. Fechner, ${ }^{13,8}$ A. Ferrero, ${ }^{16}$ A. J. Finch,${ }^{29}$ E. Frank,${ }^{3}$ Y. Fujii,,${ }^{18} \dagger$ Y. Fukuda, ${ }^{33}$ V. Galymov,${ }^{60}$ G. L. Ganetis, ${ }^{6}$ F. C. Gannaway, ${ }^{39}$ A. Gaudin, ${ }^{53}$ A. Gendotti, ${ }^{15}$ M. A. George, ${ }^{39}$ S. Giffin, ${ }^{40}$ C. Giganti, ${ }^{19}$ K. Gilje, ${ }^{34}$ A. K. Ghosh, ${ }^{6}$ T. Golan, ${ }^{59}$ M. Goldhaber, ${ }^{6 \$}$ J. J. Gomez-Cadenas, ${ }^{20}$ S. Gomi, ${ }^{28}$ M. Gonin,,${ }^{14}$ N. Grant,${ }^{29}$ A. Grant,${ }^{46}$ P. Gumplinger, ${ }^{52}$ P. Guzowski, ${ }^{21}$ D. R. Hadley, ${ }^{56}$ A. Haesler, ${ }^{16}$ M. D. Haigh, ${ }^{36}$ K. Hamano,${ }^{52}$ C. Hansen, ${ }^{20,8}$ D. Hansen, ${ }^{38}$ T. Hara, ${ }^{27}$ P. F. Harrison, ${ }^{56}$ B. Hartfiel,${ }^{31}$ M. Hartz ${ }^{60,51}$ T. Haruyama, ${ }^{18, \dagger}$ T. Hasegawa, ${ }^{18, \dagger}$ N. C. Hastings, ${ }^{40}$ A. Hatzikoutelis, ${ }^{29}$ K. Hayashi, ${ }^{18, \dagger}$ Y. Hayato, ${ }^{49}, *$ C. Hearty, ${ }^{5, \mid}$ R. L. Helmer, ${ }^{52}$ R. Henderson, ${ }^{52}$ N. Higashi, ${ }^{18, \dagger}$ J. Hignight, ${ }^{34}$ A. Hillairet, ${ }^{53}$ T. Hiraki, ${ }^{28}$ E. Hirose,${ }^{18, \dagger}$ J. Holeczek, ${ }^{45}$ S. Horikawa,${ }^{15}$ K. Huang, ${ }^{28}$ A. Hyndman, ${ }^{39}$ A. K. Ichikawa, ${ }^{28}$ K. Ieki,${ }^{28}$ M. Ieva, ${ }^{19}$ M. Iida, ${ }^{18, \dagger}$ M. Ikeda, ${ }^{28} \mathrm{~J}$. Ilic, ${ }^{47} \mathrm{~J}$. Imber, ${ }^{34} \mathrm{~T}$. Ishida, ${ }^{18, \dagger} \mathrm{C}$. Ishihara, ${ }^{50} \mathrm{~T}$. Ishii, ${ }^{18, \dagger} \mathrm{S}$. J. Ives, ${ }^{21} \mathrm{M}$. Iwasaki, ${ }^{48} \mathrm{~K}$. Iyogi, ${ }^{49}$ A. Izmaylov,${ }^{26}$ B. Jamieson, ${ }^{58}$ R. A. Johnson, ${ }^{10}$ K. K. Joo, ${ }^{9}$ G. V. Jover-Manas, ${ }^{19}$ C. K. Jung, ${ }^{34}$ H. Kaji ${ }^{50, *}$ T. Kajita, ${ }^{50 *}$ H. Kakuno, ${ }^{48}$ J. Kameda, ${ }^{49, *}$ K. Kaneyuki, ${ }^{50,}$ D. Karlen,${ }^{53,52}$ K. Kasami, ${ }^{18, \dagger}$ I. Kato,${ }^{52}$ H. Kawamuko, ${ }^{28}$ E. Kearns, ${ }^{4, *}$ M. Khabibullin, ${ }^{26}$ F. Khanam, ${ }^{11}$ A. Khotjantsev, ${ }^{26}$ D. Kielczewska, ${ }^{54}$ T. Kikawa, ${ }^{28}$ J. Kim ${ }^{5}$ J. Y. Kim, ${ }^{9}$ S. B. Kim, ${ }^{43}$ N. Kimura, ${ }^{18, \dagger}$ B. Kirby, ${ }^{5}$

J. Kisiel,${ }^{45}$ P. Kitching, ${ }^{1}$ T. Kobayashi, ${ }^{18, \dagger}$ G. Kogan, ${ }^{21}$ S. Koike, ${ }^{18, \dagger}$ A. Konaka, ${ }^{52}$ L. L. Kormos, ${ }^{29}$ A. Korzenev, ${ }^{16}$ K. Koseki, ${ }^{18}{ }^{\dagger}$ Y. Koshio, ${ }^{49}, *$ Y. Kouzuma, ${ }^{49}$ K. Kowalik, ${ }^{2}$ V. Kravtsov, ${ }^{11}$ I. Kreslo, ${ }^{3}$ W. Kropp ${ }^{7}$ H. Kubo, ${ }^{28}$ J. Kubota, ${ }^{28}$ Y. Kudenko, ${ }^{26}$ N. Kulkarni, ${ }^{31}$ Y. Kurimoto, ${ }^{28}$ R. Kurjata, ${ }^{55}$ T. Kutter, ${ }^{31}$ J. Lagoda, ${ }^{2}$ K. Laihem, ${ }^{42}$ M. Laveder, ${ }^{24}$ M. Lawe, ${ }^{44}$ K. P. Lee, ${ }^{50}$ P. T. Le,${ }^{34}$ J. M. Levy, ${ }^{37}$ C. Licciardi ${ }^{40}$ I. T. Lim, ${ }^{9}$ T. Lindner, ${ }^{5}$ C. Lister, ${ }^{56}$ R. P. Litchfield,${ }^{56,28}$ M. Litos, ${ }^{4}$

A. Longhin, ${ }^{8}$ G. D. Lopez, ${ }^{34}$ P. F. Loverre, ${ }^{25}$ L. Ludovici, ${ }^{25}$ T. Lux, ${ }^{19}$ M. Macaire, ${ }^{8}$ L. Magaletti, ${ }^{22}$ K. Mahn, ${ }^{52}$

Y. Makida, ${ }^{18, \dagger}$ M. Malek, ${ }^{21}$ S. Manly, ${ }^{41}$ A. Marchionni, ${ }^{15}$ A. D. Marino, ${ }^{10}$ A. J. Marone, ${ }^{6}$ J. Marteau, ${ }^{32}$ J. F. Martin,,${ }^{51, \|}$

T. Maruyama ${ }^{18, \dagger}$ T. Maryon, ${ }^{29}$ J. Marzec, ${ }^{55}$ P. Masliah, ${ }^{21}$ E. L. Mathie, ${ }^{40}$ C. Matsumura ${ }^{35}$ K. Matsuoka ${ }^{28}$ V. Matveev, ${ }^{26}$

K. Mavrokoridis, ${ }^{30}$ E. Mazzucato, ${ }^{8}$ N. McCauley, ${ }^{30}$ K. S. McFarland,${ }^{41}$ C. McGrew, ${ }^{34}$ T. McLachlan, ${ }^{50}$ M. Messina, ${ }^{3}$ W. Metcalf,${ }^{31}$ C. Metelko, ${ }^{47}$ M. Mezzetto, ${ }^{24}$ P. Mijakowski, ${ }^{2}$ C. A. Miller, ${ }^{52}$ A. Minamino, ${ }^{28}$ O. Mineev, ${ }^{26}$ S. Mine, ${ }^{7}$ A. D. Missert ${ }^{10}$ G. Mituka, ${ }^{50}$ M. Miura, ${ }^{49}, *$ K. Mizouchi,${ }^{52}$ L. Monfregola, ${ }^{20}$ F. Moreau, ${ }^{14}$ B. Morgan, ${ }^{56}$ S. Moriyama, ${ }^{49}, *$ A. Muir, ${ }^{46}$ A. Murakami, ${ }^{28}$ J. F. Muratore, ${ }^{6}$ M. Murdoch,${ }^{30}$ S. Murphy, ${ }^{16}$ J. Myslik, ${ }^{53}$ N. Nagai, ${ }^{28}$ T. Nakadaira, ${ }^{18,+}$ M. Nakahata, ${ }^{49, *}$ T. Nakai ${ }^{35}$ K. Nakajima, ${ }^{35}$ T. Nakamoto, ${ }^{18, \dagger}$ K. Nakamura, ${ }^{18, \pi}$ S. Nakayama, ${ }^{49, *}$ T. Nakaya ${ }^{28, *}$ D. Naples, ${ }^{38}$ M. L. Navin, ${ }^{44}$ T. C. Nicholls,${ }^{47}$ B. Nielsen, ${ }^{34}$ C. Nielsen, ${ }^{5}$ K. Nishikawa, ${ }^{18, \dagger}$ H. Nishino,${ }^{50}$ K. Nitta, ${ }^{28}$ T. Nobuhara, ${ }^{28}$ J. A. Nowak, ${ }^{31}$ Y. Obayashi, ${ }^{49, *}$ T. Ogitsu, ${ }^{18, \dagger}$ H. Ohhata, ${ }^{18, \dagger}$ T. Okamura, ${ }^{18, \dagger}$ K. Okumura, ${ }^{50, *}$ T. Okusawa, ${ }^{35}$ S. M. Oser,${ }^{5}$ M. Otani, ${ }^{28}$ R. A. Owen, ${ }^{39}$ Y. Oyama, ${ }^{18, \dagger}$ T. Ozaki, ${ }^{35}$ M. Y. Pac, ${ }^{12}$ V. Palladino, ${ }^{23}$ V. Paolone, ${ }^{38}$

P. Paul,${ }^{34}$ D. Payne,${ }^{30}$ G. F. Pearce, ${ }^{47}$ J. D. Perkin, ${ }^{44}$ V. Pettinacci, ${ }^{15}$ F. Pierre, ${ }^{8, *}$ E. Poplawska,${ }^{39}$ B. Popov, ${ }^{37, * *}$

M. Posiadala, ${ }^{54}$ J.-M. Poutissou, ${ }^{52}$ R. Poutissou, ${ }^{52}$ P. Przewlocki, ${ }^{2}$ W. Qian, ${ }^{47}$ J. L. Raaf, ${ }^{4}$ E. Radicioni, ${ }^{22}$ P. N. Ratoff, ${ }^{29}$

T. M. Raufer, ${ }^{47}$ M. Ravonel, ${ }^{16}$ M. Raymond, ${ }^{21}$ M. Reeves,${ }^{29}$ F. Retiere, ${ }^{52}$ A. Robert, ${ }^{37}$ P. A. Rodrigues, ${ }^{41}$ E. Rondio, ${ }^{2}$ J. M. Roney ${ }^{53}$ B. Rossi, ${ }^{3}$ S. Roth, ${ }^{42}$ A. Rubbia,${ }^{15}$ D. Ruterbories, ${ }^{11}$ S. Sabouri, ${ }^{5}$ R. Sacco,${ }^{39}$ K. Sakashita,${ }^{18, \dagger}$ F. Sánchez, ${ }^{19}$

A. Sarrat,${ }^{8}$ K. Sasaki, ${ }^{18, \dagger}$ K. Scholberg, ${ }^{13, *}$ J. Schwehr, ${ }^{11}$ M. Scott, ${ }^{21}$ D. I. Scully, ${ }^{56}$ Y. Seiya, ${ }^{35}$ T. Sekiguchi, ${ }^{18, \dagger}$

H. Sekiya, ${ }^{49, *}$ M. Shibata, ${ }^{18, \dagger}$ Y. Shimizu, ${ }^{50}$ M. Shiozawa, ${ }^{49}, *$ S. Short,${ }^{21}$ P. D. Sinclair, ${ }^{21}$ M. Siyad ${ }^{47}$ B. M. Smith, ${ }^{21}$ R. J. Smith,${ }^{36}$ M. Smy, ${ }^{7, *}$ J. T. Sobczyk,${ }^{59}$ H. Sobel, ${ }^{7 *}$ M. Sorel,${ }^{20}$ A. Stahl, ${ }^{42}$ P. Stamoulis, ${ }^{20}$ J. Steinmann, ${ }^{42}$ B. Still, ${ }^{39}$ J. Stone,${ }^{4, *}$ C. Strabel, ${ }^{15}$ R. Sulej, ${ }^{2}$ A. Suzuki ${ }^{27}$ K. Suzuki, ${ }^{28}$ S. Suzuki, ${ }^{18, \dagger}$ S. Y. Suzuki,${ }^{18, \dagger}$ Y. Suzuki,${ }^{18, \dagger}$ Y. Suzuki, ${ }^{49, *}$ T. Szeglowski ${ }^{45}$ M. Szeptycka, ${ }^{2}$ R. Tacik,${ }^{40,52}$ M. Tada, ${ }^{18,}$ M. Taguchi, ${ }^{28}$ S. Takahashi, ${ }^{28}$ A. Takeda, ${ }^{49, *}$ Y. Takenaga, ${ }^{49}$ Y. Takeuchi, ${ }^{27, *}$ K. Tanaka, ${ }^{18, \dagger}$ H. A. Tanaka,${ }^{5, \|}$ M. Tanaka, ${ }^{18, \dagger}$ M. M. Tanaka, ${ }^{18, \dagger}$ N. Tanimoto, ${ }^{50}$ K. Tashiro, ${ }^{35}$ I. Taylor, ${ }^{34}$

A. Terashima, ${ }^{18, \dagger}$ D. Terhorst, ${ }^{42}$ R. Terri, ${ }^{39}$ L. F. Thompson, ${ }^{44}$ A. Thorley,${ }^{30} \mathrm{~W}$. Toki, ${ }^{11}$ S. Tobayama, ${ }^{5}$ T. Tomaru, ${ }^{18, \dagger}$ Y. Totsuka, ${ }^{18,} \mathrm{C}$. Touramanis,${ }^{30} \mathrm{~T}$. Tsukamoto, ${ }^{18, \dagger} \mathrm{M}$. Tzanov,${ }^{31,10}$ Y. Uchida, ${ }^{21} \mathrm{~K}$. Ueno, ${ }^{49}$ A. Vacheret, ${ }^{21} \mathrm{M}$. Vagins, ${ }^{7, *}$ 
G. Vasseur, ${ }^{8}$ O. Veledar, ${ }^{44}$ T. Wachala, ${ }^{17}$ J. J. Walding, ${ }^{21}$ A. V. Waldron, ${ }^{36}$ C. W. Walter, ${ }^{13}$, ${ }^{*}$ P. J. Wanderer, ${ }^{6}$ J. Wang, ${ }^{48}$ M. A. Ward, ${ }^{44}$ G. P. Ward, ${ }^{44}$ D. Wark, ${ }^{47,21}$ M. O. Wascko, ${ }^{21}$ A. Weber,${ }^{36,47}$ R. Wendell, ${ }^{13}$ N. West,${ }^{36}$ L. H. Whitehead, ${ }^{56}$ G. Wikström, ${ }^{16}$ R. J. Wilkes, ${ }^{57}$ M. J. Wilking, ${ }^{52}$ Z. Williamson, ${ }^{36}$ J. R. Wilson,${ }^{39}$ R. J. Wilson, ${ }^{11}$ T. Wongjirad, ${ }^{13}$ S. Yamada, ${ }^{49}$ Y. Yamada, ${ }^{18, \dagger}$ A. Yamamoto, ${ }^{18, \dagger}$ K. Yamamoto, ${ }^{35}$ Y. Yamanoi,${ }^{18, *}$ H. Yamaoka, ${ }^{18}$ T. Yamauchi, ${ }^{28}$ C. Yanagisawa, ${ }^{34, \dagger}$ T. Yano, ${ }^{27}$ S. Yen ${ }^{52}$ N. Yershov ${ }^{26}$ M. Yokoyama, ${ }^{48, *}$ T. Yuan, ${ }^{10}$ A. Zalewska, ${ }^{17}$ J. Zalipska, ${ }^{5}$ L. Zambelli, ${ }^{37}$ K. Zaremba, ${ }^{55}$ M. Ziembicki, ${ }^{55}$ E. D. Zimmerman, ${ }^{10}$ M. Zito, ${ }^{8}$ and J. Żmuda ${ }^{59}$

(The T2K Collaboration)

\footnotetext{
${ }^{1}$ University of Alberta, Centre for Particle Physics, Department of Physics, Edmonton, Alberta, Canada

${ }^{2}$ National Center for Nuclear Research, Warsaw, Poland

${ }^{3}$ University of Bern, Albert Einstein Center for Fundamental Physics, Laboratory for High Energy Physics (LHEP), Bern, Switzerland

${ }^{4}$ Boston University, Department of Physics, Boston, Massachusetts, USA

${ }^{5}$ University of British Columbia, Department of Physics and Astronomy, Vancouver, British Columbia, Canada

${ }^{6}$ Brookhaven National Laboratory, Physics Department, Upton, New York, USA

${ }^{7}$ University of California, Irvine, Department of Physics and Astronomy, Irvine, California, USA

${ }^{8}$ IRFU, CEA Saclay, Gif-sur-Yvette, France

${ }^{9}$ Chonnam National University, Institute for Universe \& Elementary Particles, Gwangju, Korea

${ }^{10}$ University of Colorado at Boulder, Department of Physics, Boulder, Colorado, USA

${ }^{11}$ Colorado State University, Department of Physics, Fort Collins, Colorado, USA

${ }^{12}$ Dongshin University, Department of Physics, Naju, Korea

${ }^{13}$ Duke University, Department of Physics, Durham, North Carolina, USA

${ }^{14}$ Ecole Polytechnique, IN2P3-CNRS, Laboratoire Leprince-Ringuet, Palaiseau, France

${ }^{15}$ ETH Zurich, Institute for Particle Physics, Zurich, Switzerland

${ }^{16}$ University of Geneva, Section de Physique, DPNC, Geneva, Switzerland

${ }^{17}$ H. Niewodniczanski Institute of Nuclear Physics PAN, Cracow, Poland

${ }^{18}$ High Energy Accelerator Research Organization (KEK), Tsukuba, Ibaraki, Japan

${ }^{19}$ Institut de Fisica d'Altes Energies (IFAE), Bellaterra (Barcelona), Spain

${ }^{20}$ IFIC (CSIC \& University of Valencia), Valencia, Spain

${ }^{21}$ Imperial College London, Department of Physics, London, United Kingdom

${ }^{22}$ INFN Sezione di Bari and Università e Politecnico di Bari, Dipartimento Interuniversitario di Fisica, Bari, Italy

${ }^{23}$ INFN Sezione di Napoli and Università di Napoli, Dipartimento di Fisica, Napoli, Italy

${ }^{24}$ INFN Sezione di Padova and Università di Padova, Dipartimento di Fisica, Padova, Italy

${ }^{25}$ INFN Sezione di Roma and Università di Roma "La Sapienza", Roma, Italy

${ }^{26}$ Institute for Nuclear Research of the Russian Academy of Sciences, Moscow, Russia

${ }^{27}$ Kobe University, Kobe, Japan

${ }^{28}$ Kyoto University, Department of Physics, Kyoto, Japan

${ }^{29}$ Lancaster University, Physics Department, Lancaster, United Kingdom

${ }^{30}$ University of Liverpool, Department of Physics, Liverpool, United Kingdom

${ }^{31}$ Louisiana State University, Department of Physics and Astronomy, Baton Rouge, Louisiana, USA

${ }^{32}$ Université de Lyon, Université Claude Bernard Lyon 1, IPN Lyon (IN2P3), Villeurbanne, France

${ }^{33}$ Miyagi University of Education, Department of Physics, Sendai, Japan

${ }^{34}$ State University of New York at Stony Brook, Department of Physics and Astronomy, Stony Brook, New York, USA

${ }^{35}$ Osaka City University, Department of Physics, Osaka, Japan

${ }^{36}$ Oxford University, Department of Physics, Oxford, United Kingdom

${ }^{37}$ UPMC, Université Paris Diderot, CNRS/IN2P3, Laboratoire de Physique Nucléaire et de Hautes Energies (LPNHE), Paris, France

${ }^{38}$ University of Pittsburgh, Department of Physics and Astronomy, Pittsburgh, Pennsylvania, USA

${ }^{39}$ Queen Mary, University of London, School of Physics and Astronomy, London, United Kingdom

${ }^{40}$ University of Regina, Department of Physics, Regina, Saskatchewan, Canada

${ }^{41}$ University of Rochester, Department of Physics and Astronomy, Rochester, New York, USA

${ }^{42}$ RWTH Aachen University, III. Physikalisches Institut, Aachen, Germany

${ }^{43}$ Seoul National University, Department of Physics and Astronomy, Seoul, Korea

${ }^{44}$ University of Sheffield, Department of Physics and Astronomy, Sheffield, United Kingdom

${ }^{45}$ University of Silesia, Institute of Physics, Katowice, Poland

${ }^{46}$ STFC, Daresbury Laboratory, Warrington, United Kingdom

${ }^{47}$ STFC, Rutherford Appleton Laboratory, Harwell Oxford, United Kingdom

${ }^{48}$ University of Tokyo, Department of Physics, Tokyo, Japan

${ }^{49}$ University of Tokyo, Institute for Cosmic Ray Research, Kamioka Observatory, Kamioka, Japan

${ }^{50}$ University of Tokyo, Institute for Cosmic Ray Research, Research Center for Cosmic Neutrinos, Kashiwa, Japan
} 


\author{
${ }^{51}$ University of Toronto, Department of Physics, Toronto, Ontario, Canada \\ ${ }^{52}$ TRIUMF, Vancouver, British Columbia, Canada \\ ${ }^{53}$ University of Victoria, Department of Physics and Astronomy, Victoria, British Columbia, Canada \\ ${ }^{54}$ University of Warsaw, Faculty of Physics, Warsaw, Poland \\ ${ }^{55}$ Warsaw University of Technology, Institute of Radioelectronics, Warsaw, Poland \\ ${ }^{56}$ University of Warwick, Department of Physics, Coventry, United Kingdom \\ ${ }^{57}$ University of Washington, Department of Physics, Seattle, Washington, USA \\ ${ }^{58}$ University of Winnipeg, Department of Physics, Winnipeg, Manitoba, Canada \\ ${ }^{59}$ Wroclaw University, Faculty of Physics and Astronomy, Wroclaw, Poland \\ ${ }^{60}$ York University, Department of Physics and Astronomy, Toronto, Ontario, Canada
}

(Received 6 January 2012; published 22 February 2012)

\begin{abstract}
We report a measurement of muon-neutrino disappearance in the T2K experiment. The 295-km muonneutrino beam from Tokai to Kamioka is the first implementation of the off-axis technique in a longbaseline neutrino oscillation experiment. With data corresponding to $1.43 \times 10^{20}$ protons on target, we observe 31 fully-contained single $\mu$-like ring events in Super-Kamiokande, compared with an expectation of $104 \pm 14$ (syst) events without neutrino oscillations. The best-fit point for two-flavor $\nu_{\mu} \rightarrow \nu_{\tau}$ oscillations is $\sin ^{2}\left(2 \theta_{23}\right)=0.98$ and $\left|\Delta m_{32}^{2}\right|=2.65 \times 10^{-3} \mathrm{eV}^{2}$. The boundary of the $90 \%$ confidence region includes the points $\left(\sin ^{2}\left(2 \theta_{23}\right),\left|\Delta m_{32}^{2}\right|\right)=\left(1.0,3.1 \times 10^{-3} \mathrm{eV}^{2}\right),\left(0.84,2.65 \times 10^{-3} \mathrm{eV}^{2}\right)$ and $\left(1.0,2.2 \times 10^{-3} \mathrm{eV}^{2}\right)$.
\end{abstract}

DOI: 10.1103/PhysRevD.85.031103

We report a measurement of muon-neutrino disappearance in the T2K experiment. The muon-neutrino beam from Tokai to Kamioka is the first implementation of the off-axis technique [1] in a long-baseline neutrino oscillation experiment. The off-axis technique is used to provide a narrow-band neutrino energy spectrum tuned to the value of $L / E$ that maximizes the neutrino oscillation effect due to $\Delta m_{32}^{2}$, the mass splitting first observed in atmospheric neutrinos [2]. This narrow-band energy spectrum also provides a clean signature for subdominant electron neutrino appearance, as we have recently reported [3]. Muonneutrino disappearance depends on the survival probability, which, in the framework of two-flavor $\nu_{\mu} \rightarrow \nu_{\tau}$ oscillations, is given by

$$
P_{\text {surv }}=1-\sin ^{2}\left(2 \theta_{23}\right) \sin ^{2}\left(\frac{\Delta m_{32}^{2} L}{4 E}\right),
$$

where $E$ is the neutrino energy and $L$ is the neutrino propagation length. We have neglected subleading oscillation terms. In this paper, we describe our observation of $\nu_{\mu}$ disappearance, and we use the result to measure $\left|\Delta m_{32}^{2}\right|$ and $\sin ^{2}\left(2 \theta_{23}\right)$. Previous measurements of these neutrino mixing parameters have been reported by $\mathrm{K} 2 \mathrm{~K}$ [4] and MINOS [5], which use on-axis neutrino beams, and SuperKamiokande [6], which uses atmospheric neutrinos.

\footnotetext{
*Also at IPMU, TODIAS, Univ. of Tokyo, Japan

${ }^{\dagger}$ Also at J-PARC Center

Deceased

${ }^{\S}$ Now at CERN

"Also at Institute of Particle Physics, Canada

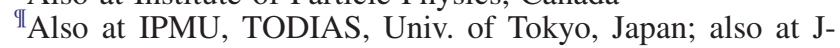
PARC Center

**Also at JINR, Dubna, Russia

${ }^{\dagger}$ Also at BMCC/CUNY, NY, NY, USA
}

PACS numbers: 14.60.Pq, 13.15.+g, 25.30.Pt, 95.55.Vj

Details of the T2K experimental setup are described elsewhere [7]. Here, we briefly review the components relevant for the $\nu_{\mu}$ oscillation analysis. The J-PARC Main Ring accelerator [8] provides $30 \mathrm{GeV}$ protons with a cycle of $0.3 \mathrm{~Hz}$. Six bunches (Run 1) or eight bunches (Run 2) are extracted in a 5- $\mu$ s spill and are transported to the production target through an arc instrumented by superconducting magnets. The proton beam position, profile, timing and intensity are measured by 21 electrostatic beam position monitors, 19 segmented secondary emission monitors, one optical transition radiation monitor and five current transformers. The secondary beam line, filled with helium at atmospheric pressure, is composed of the target, focusing horns and decay tunnel. The graphite target is $2.6 \mathrm{~cm}$ in diameter and $90 \mathrm{~cm}\left(1.9 \lambda_{\text {int }}\right)$ long. Positivelycharged particles exiting the target are focused into the 96 m-long decay tunnel by three magnetic horns pulsed at $250 \mathrm{kA}$. Neutrinos are primarily produced in the decays of charged pions and kaons. A beam dump is located at the end of the tunnel and is followed by muon monitors measuring the beam direction of each spill.

The neutrino beam is directed $2.5^{\circ}$ off the axis between the target and the Super-Kamiokande (SK) far detector $295 \mathrm{~km}$ away. This configuration produces a narrow-band $\nu_{\mu}$ beam with peak energy tuned to the first oscillation maximum $E_{\nu}=\left|\Delta m_{32}^{2}\right| L /(2 \pi) \simeq 0.6 \mathrm{GeV}$.

The near-detector complex (ND280) [7] is located $280 \mathrm{~m}$ downstream from the target and hosts two detectors. The on-axis Interactive Neutrino GRID [9] records neutrino interactions with high statistics to monitor the beam intensity, direction and profile. It consists of 14 identical 7 ton modules composed of an iron-absorber/scintillatortracker sandwich arranged in $10 \mathrm{~m}$ by $10 \mathrm{~m}$ crossed horizontal and vertical arrays centered on the beam. The 


\section{K. ABE et al.}

off-axis detector reconstructs exclusive final states to study neutrino interactions and beam properties corresponding to those expected at the far detector. Embedded in the refurbished UA1/NOMAD magnet (field strength $0.2 \mathrm{~T}$ ), it consists of three large-volume time projection chambers (TPCs) [10] interleaved with two fine-grained tracking detectors (FGDs, each 1 ton). It also has a $\pi^{0}$-optimized detector and a surrounding electromagnetic calorimeter. The magnet yoke is instrumented as a side muon range detector.

The SK water-Cherenkov far detector [11] has a fiducial volume of $22.5 \mathrm{kt}$ within its cylindrical inner detector (ID). Enclosing the ID is the $2 \mathrm{~m}$-wide outer detector (OD). The front-end readout electronics [7] allow for a dead-time-free trigger. Spill timing information, synchronized by the global positioning system with $<150 \mathrm{~ns}$ precision, is transferred from J-PARC to SK and triggers the recording of photomultiplier hits within $\pm 500 \mu$ s of the expected neutrino arrival time.

The results presented in this paper are based on the first two physics runs: Run 1 (January-June 2010) and Run 2 (November 2010-March 2011). During this time period, the Main Ring proton beam power was continually increased and reached $145 \mathrm{~kW}$ with $9 \times 10^{13}$ protons per pulse. The fraction of protons hitting the target was monitored by the electrostatic beam position monitors, segmented secondary emission monitors and optical transition radiation monitor and found to be greater than $99 \%$ and stable in time. A total of 2, 474, 419 spills was retained for analysis after beam and far-detector quality cuts, corresponding to $1.43 \times 10^{20}$ protons on target (POT).

We present the study of events in the far detector with a single muonlike ( $\mu$-like) ring. The event selection enhances $\nu_{\mu}$ charged-current quasielastic interactions (CCQE). For these events, neglecting the Fermi motion, the neutrino energy $E_{\nu}$ can be reconstructed as

$$
E_{\nu}=\frac{m_{p}^{2}-\left(m_{n}-E_{b}\right)^{2}-m_{\mu}^{2}+2\left(m_{n}-E_{b}\right) E_{\mu}}{2\left(m_{n}-E_{b}-E_{\mu}+p_{\mu} \cos \theta_{\mu}\right)}
$$

where $m_{p}$ is the proton mass, $m_{n}$ the neutron mass, and $E_{b}=27 \mathrm{MeV}$ is the binding energy of a nucleon inside a ${ }^{16} \mathrm{O}$ nucleus. In Eq. (2), $E_{\mu}, p_{\mu}$ and $\theta_{\mu}$ are, respectively, the measured muon energy, momentum and angle with respect to the incoming neutrino. The selection criteria for this analysis were fixed from Monte Carlo (MC) studies before the data were collected. The observed number of events and spectrum are compared with signal and background expectations, which are based on neutrino flux and cross-section predictions and are corrected using an inclusive measurement in the off-axis near detector.

Our predicted beam flux (Fig. 1) is based on models tuned to experimental data. The most significant constraint comes from NA61 measurements of pion production [12] in $(p, \theta)$ bins, where $p$ is the pion momentum and $\theta$ the
PHYSICAL REVIEW D 85, 031103(R) (2012)

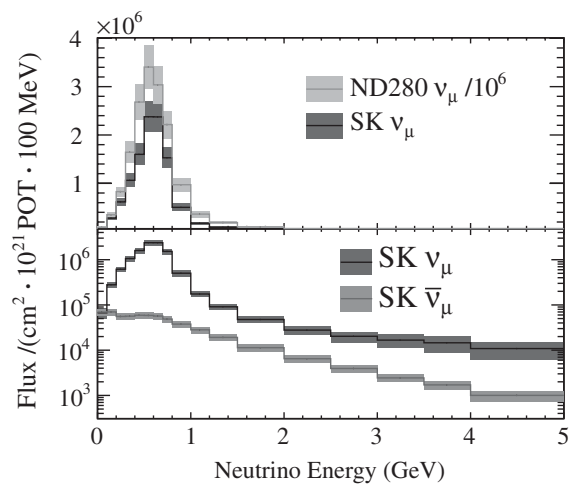

FIG. 1. (Top) the predicted flux of $\nu_{\mu}$ as a function of neutrino energy without oscillations at Super-Kamiokande and at the offaxis near detector; (bottom) the flux of $\nu_{\mu}$ and $\bar{\nu}_{\mu}$ at SuperKamiokande. The shaded boxes indicate the total systematic uncertainty for each energy bin.

polar angle with respect to the proton beam; there are $5 \%-10 \%$ systematic and similar statistical uncertainties in most of the measured phase space. The production of pions in the target outside the NA61-measured phase space and all kaon production are modeled using FLUKA $[13,14]$. The production rate of these pions is assigned systematic uncertainties of $50 \%$, and kaon production uncertanties are estimated to be between $15 \%$ and $100 \%$ based on a comparison of FLUKA with data from Eichten et al. [15]. The software package GEANT3 [16], with GCALOR [17] for hadronic interactions, handles particle propagation through the magnetic horns, target hall, decay volume and beam dump. Additional systematic errors in the neutrino fluxes are included for uncertainties in secondary nucleon production and total hadronic inelastic cross sections, uncertainties in the proton beam direction, spatial extent and angular divergence, the horn current, and the secondary beam line component alignment uncertainties. The stability of the beam direction and neutrino rate per proton on target are monitored continuously with Interactive Neutrino GRID and are within the assigned systematic uncertainties [3].

Systematic uncertainties in the shape of the flux as a function of neutrino energy require knowledge of the correlations of the uncertainties in $(p, \theta)$ bins of hadron production. For the NA61 pion-production data [12], we assume full correlation between $(p, \theta)$ bins for each individual source of systematic uncertainty, except for particle identification where there is a known momentum-dependent correlation. Where correlations of hadron-production uncertainties are unknown, we choose correlations in kinematic variables to maximize the uncertainty in the normalization of the predicted flux.

Neutrino interactions are simulated using the NEUT event generator [18]. Uncertainties in cross sections of the exclusive neutrino processes are determined by comparisons with recent measurements from the SciBooNE 


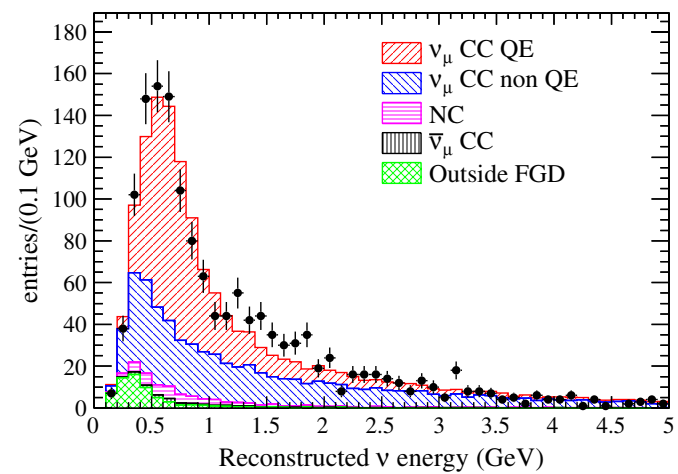

FIG. 2 (color online). Neutrino energy reconstructed for the CCQE hypothesis for $\nu_{\mu} \mathrm{CC}$ candidates interacting in the FGD target. The data are shown using points with error bars (statistical only), and the MC predictions are in shaded histograms.

[19], MiniBooNE [20,21] and K2K [22,23] experiments, comparisons with the GENIE [24] and NuWro [25] generators and recent theoretical work [26].

An inclusive $\nu_{\mu}$ charged-current (CC) measurement in the off-axis near detector (ND) is used to constrain the expected event rate at the far detector. From a data sample collected in Run 1 of $2.88 \times 10^{19}$ POT, neutrino interactions are selected in the FGDs with charged particles entering the downstream TPC. The most energetic negatively charged particle in the TPC is required to have ionization energy loss compatible with that of a muon. The analysis selects 1529 data events with $38 \% \nu_{\mu}$ CC efficiency and 90\% purity. The agreement between the reconstructed neutrino energy in data and MC is shown in Fig. 2. The ratio of measured $\nu_{\mu} \mathrm{CC}$ interactions to $\mathrm{MC}$ is

$$
\begin{aligned}
R_{\mathrm{ND}}^{\nu_{\mu} \mathrm{CC}}= & \frac{N_{\mathrm{ND}}^{\mathrm{Data}, \nu_{\mu} \mathrm{CC}}}{N_{\mathrm{ND}}^{\mathrm{MC}, \nu_{\mu} \mathrm{CC}}} \\
= & 1.036 \pm 0.028 \text { (stat. })_{-0.037}^{+0.044} \text { (det. syst.) } \\
& \pm 0.038 \text { (phys. syst.), }
\end{aligned}
$$

where $N_{\mathrm{ND}}^{\mathrm{Data}, \nu_{\mu} \mathrm{CC}}$ is the number of $\nu_{\mu} \mathrm{CC}$ events, and $N_{\mathrm{ND}}^{\mathrm{MC}, \nu_{\mu} \mathrm{CC}}$ is the MC prediction normalized by POT. The detector systematic errors in Eq. (3) are mainly due to uncertainties in tracking and particle identification efficiencies. The physics uncertainties result from crosssection uncertainties but exclude normalization uncertainties that cancel in a far/near ratio.

At the far detector, we select a $\nu_{\mu}$ CCQE-enriched sample. The SK event reconstruction [27] uses PMT hits in time with a neutrino spill. We select a fully contained fiducial volume sample by requiring no activity in the OD, no preactivity in the $100 \mu \mathrm{s}$ before the event trigger time, at least $30 \mathrm{MeV}$ electron-equivalent energy deposited in the ID and a reconstructed event vertex in the fiducial region. The OD veto rejects events induced by neutrino interactions outside of the ID and events where energy escapes from the ID. The visible energy requirement rejects events from radioactive decays in the detector. The fiducial vertex requirement rejects particles entering from outside the ID. Further conditions are required to enrich the sample in $\nu_{\mu}$ CCQE events: a single Cherenkov ring identified as a muon, with momentum $p_{\mu}>200 \mathrm{MeV} / \mathrm{c}$, and no more than one delayed electron. The muon momentum requirement rejects charged pions and misidentified electrons from the decay of unseen muons and pions, and the delayed-electron veto rejects events with muons accompanied by unseen pions and muons. The number of events in data and MC after each selection criterion is shown in Table I. The efficiency and purity of $\nu_{\mu}$ CCQE events are estimated to be $72 \%$ and $61 \%$, respectively.

We calculate the expected number of signal events in the far detector $\left(N_{\mathrm{SK}}^{\exp }\right)$ by correcting the far-detector MC prediction with $R_{\mathrm{ND}}^{\nu_{\mu} \mathrm{CC}}$ from Eq. (3):

$$
N_{\mathrm{SK}}^{\mathrm{exp}}\left(E_{r}\right)=R_{\mathrm{ND}}^{\nu_{\mu} \mathrm{CC}} \sum_{E_{t}} P_{\text {surv }}\left(E_{t}\right) N_{\mathrm{SK}}^{\mathrm{MC}}\left(E_{r}, E_{t}\right) .
$$

In Eq. (4), $N_{\mathrm{SK}}^{\mathrm{MC}}\left(E_{r}, E_{t}\right)$ is the expected number of events for the no-disappearance hypothesis for T2K Runs 1 and 2 in bins of reconstructed $\left(E_{r}\right)$ and true $\left(E_{t}\right)$ energies. $P_{\text {surv }}\left(E_{t}\right)$ is the two-flavor $\nu_{\mu}$-survival probability and is applied to $\nu_{\mu}$ and $\bar{\nu}_{\mu} \mathrm{CC}$ interactions but not to neutralcurrent interactions.

TABLE I. Event reduction at the far detector. After each selection criterion is applied, the number of observed (Data) and MC expected events of $\nu_{\mu}$ CCQE, $\nu_{\mu}$ CC non-QE, intrinsic $\nu_{e}$, and neutral current (NC) are given. The columns denoted by $\nu_{\mu}$ include $\bar{\nu}_{\mu}$. All MC CC samples assume $\nu_{\mu} \rightarrow \nu_{\tau}$ oscillations with $\sin ^{2}\left(2 \theta_{23}\right)=1.0$ and $\left|\Delta m_{32}^{2}\right|=2.4 \times 10^{-3} \mathrm{eV}^{2}$.

\begin{tabular}{lccccr}
\hline \hline & Data & $\nu_{\mu} \mathrm{CCQE}$ & $\nu_{\mu} \mathrm{CC}$ non-QE & $\nu_{e} \mathrm{CC}$ & $\mathrm{NC}$ \\
\hline Fiducial Volume interaction & $\mathrm{n} / \mathrm{a}$ & 24.0 & 43.7 & 3.1 & 71.0 \\
Fully Contained Fiducial Volume & 88 & 19.0 & 33.8 & 3.0 & 18.3 \\
single ring & 41 & 17.9 & 13.1 & 1.9 & 5.7 \\
$\mu$-like & 33 & 17.6 & 12.4 & $<0.1$ & 1.9 \\
$p_{\mu}>200 \mathrm{MeV} / \mathrm{c}$ & 33 & 17.5 & 12.4 & $<0.1$ & 1.9 \\
0 or 1 delayed $e$ & 31 & 17.3 & 9.2 & $<0.1$ & 1.8 \\
\hline \hline
\end{tabular}


TABLE II. Systematic uncertainties on the predicted number of SK selected events without oscillations and for oscillations with $\sin ^{2}\left(2 \theta_{23}\right)=1.0$ and $\left|\Delta m_{32}^{2}\right|=2.4 \times 10^{-3} \mathrm{eV}^{2}$.

\begin{tabular}{lcc}
\hline \hline Source & $\delta N_{\mathrm{SK}}^{\mathrm{exp}} / N_{\mathrm{SK}}^{\mathrm{exp}}(\%$, no osc $)$ & $\delta N_{\mathrm{SK}}^{\exp } / N_{\mathrm{SK}}^{\exp }(\%$, with osc $)$ \\
\hline SK CCQE efficiency & \pm 3.4 & \pm 3.4 \\
SK CC non-QE efficiency & \pm 3.3 & \pm 6.5 \\
SK NC efficiency & \pm 2.0 & \pm 7.2 \\
ND280 efficiency & $+5.5-5.3$ & $+5.5-5.3$ \\
ND280 event rate & \pm 2.6 & \pm 2.6 \\
Flux normalization (SK/ND280) & \pm 7.3 & \pm 4.8 \\
CCQE cross section & \pm 4.1 & \pm 2.5 \\
CC1 $\pi / C C Q E$ cross section & $+2.2-1.9$ & $+0.4-0.5$ \\
Other CC/CCQE cross section & $+5.3-4.7$ & $+4.1-3.6$ \\
NC/CCQE cross section & \pm 0.8 & \pm 0.9 \\
Final-state interactions & \pm 3.2 & \pm 5.9 \\
Total & $+13.3-13.0$ & $+15.0-14.8$ \\
\hline \hline
\end{tabular}

The sources of systematic uncertainty in $N_{\mathrm{SK}}^{\mathrm{exp}}$ are listed in Table II. Uncertainties in the near-detector and fardetector selection efficiencies are energy-independent except for the ring-counting efficiency. Uncertainty in the near-detector event rate is applied to $N_{\mathrm{ND}}^{\mathrm{Data}, \nu_{\mu} \mathrm{CC}}$ in Eq. (3). The flux normalization uncertainty is reduced because of the near-detector constraint. The uncertainty in the flux shape is propagated using the covariance matrix when calculating $N_{\mathrm{SK}}^{\mathrm{exp}}$. The near-detector constraint also leads to partial cancellation in the uncertainty in cross-section modeling, but the cancellation is not complete due to the different fluxes, different acceptances and different nuclei in the near and far detectors. The total uncertainty in $N_{\mathrm{SK}}^{\exp }$ is ${ }_{-13.0 \%}^{+13.3 \%}$ without oscillations and ${ }_{-14.8 \%}^{+15.0 \%}$ with oscillations with $\sin ^{2}\left(2 \theta_{23}\right)=1.0$ and $\left|\Delta m_{32}^{2}\right|=2.4 \times 10^{-3} \mathrm{eV}^{2}$.

We find the best-fit values of the oscillation parameters using a binned likelihood-ratio method, in which $\sin ^{2}\left(2 \theta_{23}\right)$ and $\left|\Delta m_{32}^{2}\right|$ are varied in the input to the calculation of $N_{\text {SK }}^{\text {exp }}$ until

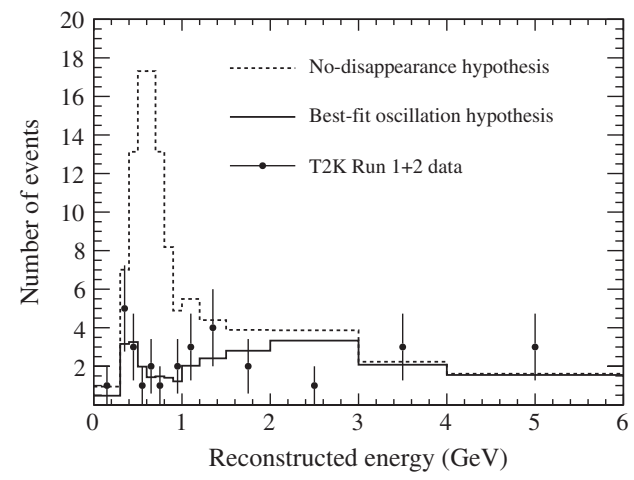

FIG. 3. Reconstructed energy spectrum of the 31 data events compared with the expected spectra in the far detector without disappearance and with best-fit $\nu_{\mu} \rightarrow \nu_{\tau}$ oscillations. A variable binning scheme is used here for the purpose of illustration only; the actual analysis used equal-sized $50 \mathrm{MeV}$ bins.

$$
2 \sum_{E_{r}}\left[N_{\mathrm{SK}}^{\mathrm{data}} \ln \left(\frac{N_{\mathrm{SK}}^{\mathrm{data}}}{N_{\mathrm{SK}}^{\exp }}\right)+\left(N_{\mathrm{SK}}^{\text {exp }}-N_{\mathrm{SK}}^{\mathrm{data}}\right)\right]
$$

is minimized. The sum in Eq. (5) is over $50 \mathrm{MeV}$ bins of reconstructed energy of selected events in the far detector from $0-10 \mathrm{GeV}$.

Using the near-detector measurement and setting $P_{\text {surv }}=1.0$ in Eq. (4), we expect a total of $103.6_{-13.4}^{+13.8}$ (systematic) single $\mu$-like ring events in the far detector without disappearance, but we observe 31 events. If $\nu_{\mu} \rightarrow \nu_{\tau}$ oscillations are assumed, the best-fit point determined using Eq. (5) is $\sin ^{2}\left(2 \theta_{23}\right)=0.98$ and $\left|\Delta m_{32}^{2}\right|=$ $2.65 \times 10^{-3} \mathrm{eV}^{2}$. We estimate the systematic uncertainty in the best-fit value of $\sin ^{2}\left(2 \theta_{23}\right)$ to be $\pm 4.7 \%$ and that in $\left|\Delta m_{32}^{2}\right|$ to be $\pm 4.5 \%$. The reconstructed energy spectrum of the 31 data events is shown in Fig. 3 along with the expected far-detector spectra without disappearance and with best-fit oscillations.

We construct confidence regions ${ }^{1}$ in the oscillation parameters using the method of Feldman and Cousins [28]. Statistical variations are taken into account by Poisson fluctuations of toy MC data sets, and systematic uncertainties are incorporated using the method of Cousins and Highland [29,30]. The 90\% confidence region for $\sin ^{2}\left(2 \theta_{23}\right)$ and $\left|\Delta m_{32}^{2}\right|$ is shown in Fig. 4 for combined statistical and systematic uncertainties.

We also carried out an alternate analysis with a maximum likelihood method. The likelihood is defined as:

$$
\begin{aligned}
L= & L_{\text {norm }}\left(\sin ^{2}\left(2 \theta_{23}\right), \Delta m_{32}^{2}, \mathbf{f}\right) \\
& \times L_{\text {shape }}\left(\sin ^{2}\left(2 \theta_{23}\right), \Delta m_{32}^{2}, \mathbf{f}\right) L_{\text {syst }}(\mathbf{f}),
\end{aligned}
$$

\footnotetext{
${ }^{1}$ In the T2K narrow-band beam, for a low-statistics data set, there is a possible degeneracy between the first oscillation maximum and other oscillation maxima in $L / E$. Therefore, we decided in advance to report confidence regions both with and without an explicit bound at $\left|\Delta m_{32}^{2}\right|<5 \times 10^{-3} \mathrm{eV}^{2}$. For this data set, the bounded and unbounded confidence regions are identical.
} 


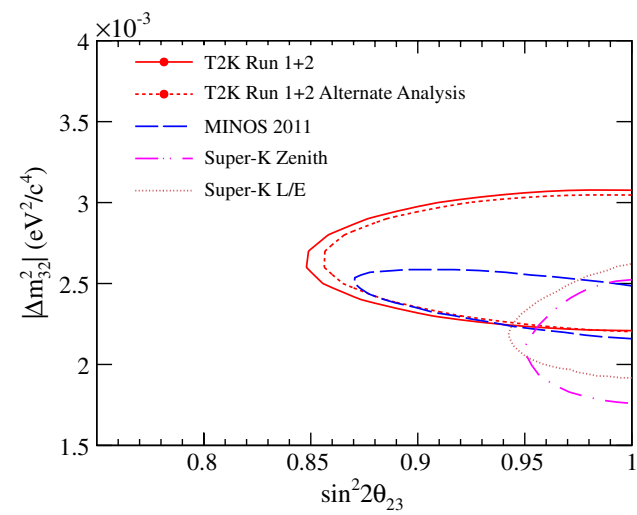

FIG. 4 (color online). The 90\% confidence regions for $\sin ^{2}\left(2 \theta_{23}\right)$ and $\left|\Delta m_{32}^{2}\right|$; results from the two analyses reported here are compared with those from MINOS [5] and SuperKamiokande [6,31].

where the first term is the Poisson probability for the observed number of events, and the second term is the unbinned likelihood for the reconstructed neutrino energy spectrum. The vector $\mathbf{f}$ represents parameters related to systematic uncertainties that have been allowed to vary in the fit to maximize the likelihood, and the last term in Eq. (6) is a multidimensional Gaussian probability for the systematic error parameters. The result is consistent with the analysis described earlier. The best-fit point for this alternate analysis is $\sin ^{2}\left(2 \theta_{23}\right)=0.99$ and $\left|\Delta m_{32}^{2}\right|=2.63 \times 10^{-3} \mathrm{eV}^{2}$. The $90 \%$ confidence region for the neutrino oscillation parameters is shown in Fig. 4.

In conclusion, we have reported the first observation of $\nu_{\mu}$ disappearance using detectors positioned off-axis in the beam of a long-baseline neutrino experiment. The values of the oscillation parameters $\sin ^{2}\left(2 \theta_{23}\right)$ and $\left|\Delta m_{32}^{2}\right|$ obtained are consistent with those reported by MINOS [5] and Super-Kamiokande [6,31].

We thank the J-PARC accelerator team for the superb accelerator performance and CERN NA61 colleagues for providing essential particle production data and for their fruitful collaboration. We acknowledge the support of MEXT, Japan; NSERC, NRC and CFI, Canada; CEA and CNRS/IN2P3, France; DFG, Germany; INFN, Italy; Ministry of Science and Higher Education, Poland; RAS, RFBR and the Ministry of Education and Science of the Russian Federation; MEST and NRF, South Korea; MICINN and CPAN, Spain; SNSF and SER, Switzerland; STFC, U.K.; NSF and DOE, USA We also thank CERN for their donation of the UA1/NOMAD magnet and DESY for the HERA-B magnet mover system. In addition, participation of individual researchers and institutions in $\mathrm{T} 2 \mathrm{~K}$ has been further supported by funds from: ERC (FP7), EU; JSPS, Japan; Royal Society, UK; DOE Early Career program, and the A.P. Sloan Foundation, USA
[1] D. Beavis et al. (E889 Collaboration), Physics Design BNL Report No. 52459, 1995.

[2] Y. Fukuda et al. (Super-Kamiokande Collaboration), Phys. Rev. Lett. 81, 1562 (1998).

[3] K. Abe et al. (T2K Collaboration), Phys. Rev. Lett. 107, 041801 (2011).

[4] M. H. Ahn et al. (K2K Collaboration), Phys. Rev. D 74, 072003 (2006).

[5] P. Adamson et al. (MINOS Collaboration), Phys. Rev. Lett. 106, 181801 (2011).

[6] K. Abe et al. (Super-Kamiokande Collaboration), Phys. Rev. Lett. 107, 241801 (2011).

[7] K. Abe et al. (T2K Collaboration), Nucl. Instrum. Methods Phys. Res., Sect. A 659, 106 (2011).

[8] Y. Yamazaki et al., KEK Report No. 2002-13 JAERI-Tech Report Nos. 2003-44, J-PARC-03-01, 2003).

[9] K. Abe et al. (T2K Collaboration), arXiv:1111.3119 [Nucl. Instrum. Meth. (to be published)].

[10] N. Abgrall et al., Nucl. Instrum. Methods Phys. Res., Sect. A 637, 25 (2011).

[11] Y. Fukuda et al. (Super-Kamiokande Collaboration), Nucl. Instrum. Methods Phys. Res., Sect. A 501, 418 (2003).

[12] N. Abgrall et al. (NA61/SHINE Collaboration), Phys. Rev. C 84, 034604 (2011).
[13] A. Ferrari, P. R. Sala, A. Fasso, and J. Ranft, CERN Report No. 2005-010, 2005.

[14] G. Battistoni et al., AIP Conf. Proc. 896, 31 (2007).

[15] T. Eichten et al., Nucl. Phys. B44, 333 (1972).

[16] R. Brun, F. Carminati, and S. Giani, CERN Report No. W5013, 1994.

[17] C. Zeitnitz and T. A. Gabriel, in International Conference on Calorimetry in High Energy Physics, Tallahasse, Florida, 1993 (unpublished).

[18] Y. Hayato, Acta Phys. Pol. B 40, 2477 (2009).

[19] J. Alcaraz-Aunion and J. Walding (SciBooNE Collaboration), AIP Conf. Proc. 1189, 145 (2009).

[20] A. A. Aguilar-Arevalo et al. (MiniBooNE Collaboration), Phys. Rev. D 81, 092005 (2010).

[21] A. A. Aguilar-Arevalo et al. (MiniBooNE Collaboration), Phys. Rev. Lett. 103, 081801 (2009).

[22] R. Gran et al. (K2K Collaboration), Phys. Rev. D 74, 052002 (2006).

[23] A. Rodriguez et al. (K2K Collaboration), Phys. Rev. D 78, 032003 (2008).

[24] C. Andreopoulos et al., Nucl. Instrum. Methods Phys. Res., Sect. A 614, 87 (2010).

[25] C. Juszczak, Acta Phys. Pol. B 40, 2507 (2009).

[26] C. Juszczak, J. T. Sobczyk, and J. Żmuda, Phys. Rev. C 82, 045502 (2010). 
[27] Y. Ashie et al. (Super-Kamiokande Collaboration), Phys. Rev. D 71, 112005 (2005).

[28] G. J. Feldman and R. D. Cousins, Phys. Rev. D 57, 3873 (1998).

[29] R. Cousins and V. Highland, Nucl. Instrum. Methods Phys. Res., Sect. A 320, 331 (1992).
[30] J. Conrad, O. Botner, A. Hallgren, and C. Perez de los Heros, Phys. Rev. D 67, 012002 (2003).

[31] Y. Takeuchi (Super-Kamiokande Collaboration), in Proceedings of Neutrino 2010, Athens, Greece, 2010 (to be published), arXiv: 1112.3425. 